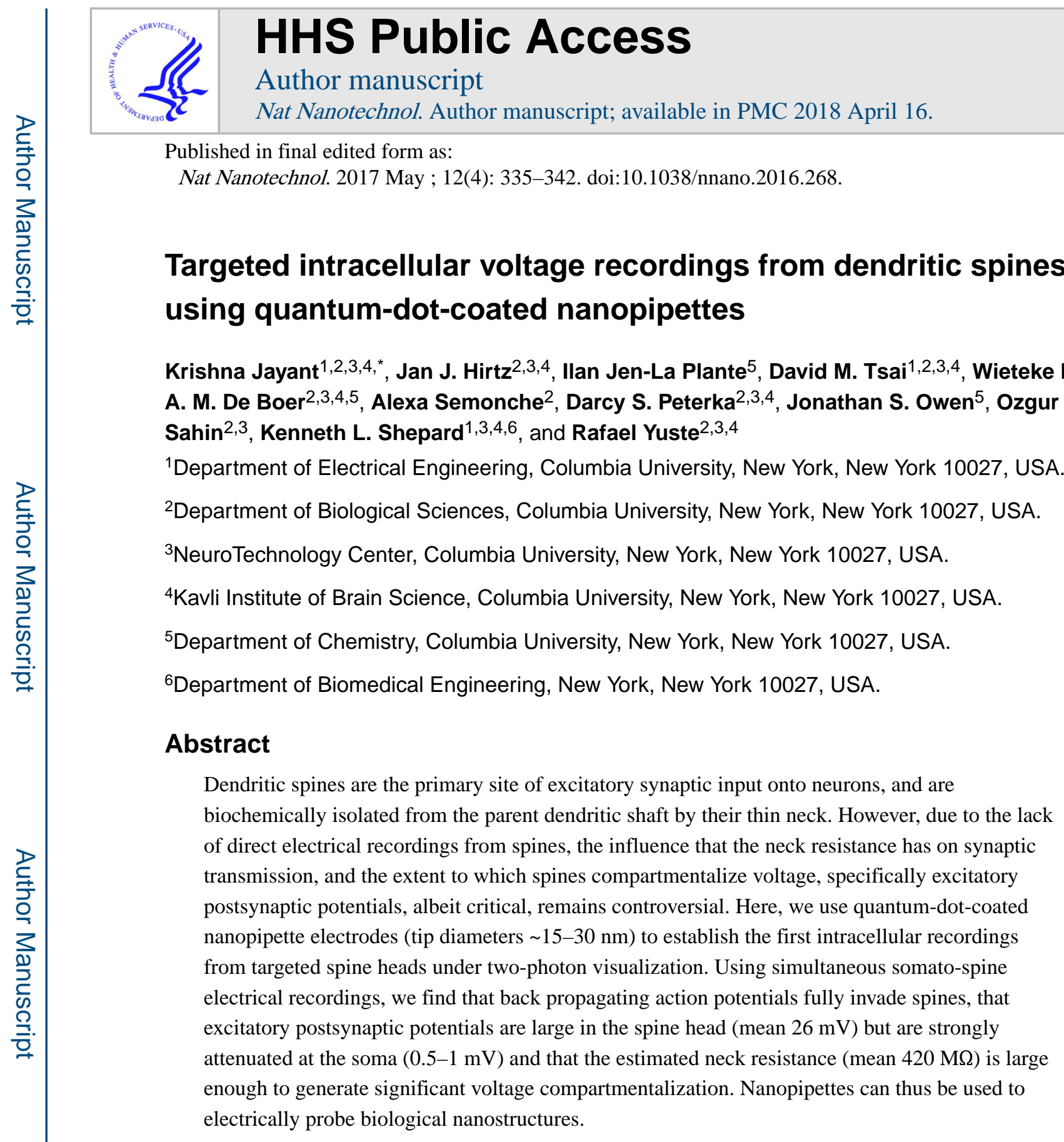

Dendritic spines, characterized by their small head (volume $\sim 0.001-0.1 \mu \mathrm{m}^{3}$ ) and narrow neck (diameter $\sim 0.1 \mu \mathrm{m}$, length $\sim 1 \mu \mathrm{m}$ ), are the primary site of excitatory synaptic input in

\footnotetext{
Reprints and permissions information is available online at www.nature.com/reprints.

*Correspondence and requests for materials should be addressed to K.J., kj2346@columbia.edu.

Author contributions

K.J. and R.Y. designed and conceptualized the study. J.J.H. performed slice preparation and provided technical support. I.J.-L.P., W.D.A.M.D.B. and J.S.O. synthesized, characterized and provided quantum dots. D.M.T. and K.J. performed SEM imaging. A.S. prepared cell cultures. D.S.P. provided technical support. O.S, K.L.S. and R.Y. advised on experiments. R.Y. supervised the overall study. K.J. performed all the experiments, modelling and analysis and wrote the manuscript. All the authors discussed the manuscript and provided comments.

Supplementary information is available in the online version of the paper.

Competing financial interests

The authors declare no competing financial interests.
} 
the cortex ${ }^{1}$. Their function and plasticity are essential for long-term potentiation ${ }^{2,3}$, brain development ${ }^{4}$ and memory formation ${ }^{5}$. Spine morphology, and, in particular, the shape and size of the spine head, are correlated with changes in synaptic strength during synaptic plasticity ${ }^{6}$. However, exactly how these morphological features influence synaptic transmission is still unclear. The spine neck forms a diffusion barrier, biochemically isolating the spine head from its parent dendritic branch ${ }^{7,8}$. Moreover, this diffusional regulation may dynamically change as a consequence of synaptic activity ${ }^{9}$. A less understood property of the spine neck is its ability to electrically influence synaptic potentials ${ }^{1,2,10-14}$. Specifically, neurotransmitter activation of receptors located on the spine head cause excitatory postsynaptic potentials (EPSPs) that trigger calcium accumulations in the spine ${ }^{7}$, and eventually propagate downstream as dendritic and somatic EPSPs ${ }^{10}$. The spine neck is believed to play a major role during this signal processing operation by filtering the EPSP as it invades the dendrite while allowing back propagating action potentials (bAPs) and dendritic signals to fully invade the spine ${ }^{15,16}$, a feature that shares remarkable similarities with the operation of semiconductor diodes. Previous studies on spine electrical properties and electrical compartmentalization have either been computational ${ }^{11,17,18}$ or indirect, through optical measurements $2,9,10,15,16,19-23$. But, even with this wealth of experimental and theoretical evidence, estimates of EPSP amplitudes, spine neck resistances $\left(R_{\text {neck }}\right)$ and basic spine biophysical mechanisms contradict each other ${ }^{10,15,18-21,24,25}$ with no clear consensus yet. A major limitation is the small size of spines, which has rendered conventional patch-clamp electrophysiology impractical. While optical measurements of voltage in spines are gaining precedence ${ }^{25}$, they still suffer from poor signal-to-noise ratio (SNR) ${ }^{16,21-24,26}$. As an alternative, and to ensure a more complete understanding of spine membrane biophysics, we explored the use of nanoscale electrodes to directly measure from the spine head.

Nanowires ${ }^{27-31}$, nanotubes ${ }^{32}$, nanopipettes ${ }^{33}$ and sharp tip electrodes ${ }^{34-37}$ have recently gained significant attention as minimally invasive intracellular electrodes and are ideally poised to revolutionize intracellular neurotechnologies ${ }^{38}$. However, only a few reports ${ }^{29,33,36,37}$ have directly demonstrated their use in intra-cellular recordings from neurons. Notably, whole-cell patch-clamp recordings from large axonal terminals and presynaptic terminals (size $\sim 2-5 \mu \mathrm{m}$ ) were recently established using high-impedance patch pipettes ${ }^{33,39}$. Although these reports present major advances in the ability to record from small compartments, their applicability to directly probe thin dendrites, axons and small synaptic structures such as dendritic spines, which together represent the majority of the structural features of all nervous systems, remains elusive. Moreover, while whole-cell electrophysiology ensures a giga-seal and sensitive voltage clamp measurements, a major limitation, especially when probing small compartments $(\sim 1 \mu \mathrm{m})$ is the washout of intracellular fluid and organelles, which can potentially lead to non-physiological conditions. Ideally, the intracellular probe and associated sensing module should be (i) extremely small to reduce membrane damage during entry, ensure tight membrane coupling and guarantee registry to subcellular structures; (ii) designed to reduce signal filtering, ensure high bandwidth and high SNR; and (iii) applicable in cultures, brain slices and in vivo preparations. In this Article, we describe and demonstrate quartz nanopipette electrodes with tip diameters between $15-30 \mathrm{~nm}$ as direct electrical interfaces to dendritic spines. 


\section{Experimental overview}

We first used neuronal cultures and identified neurons with prominent dendritic arborization. After establishing the somatic whole-cell recording, bAPs were elicited, which propagate back through the dendrite ${ }^{40},{ }^{41}$ into spines (Fig. 1a). Since bAPs are known to invade spines with very little $\operatorname{loss}^{42}$ they served as a 'control signal' standard for the nanopipette spine electrical measurement (Fig. 1b). Diffusion of a dye (Alexa-488, Alexa-594) from the patch pipette ensured two-photon visualization of dendrites and spines (Fig. 1c,d). To assist in tip localization and targeting spines, we coated the pipettes with $\mathrm{CdSe} / \mathrm{CdS} / \mathrm{ZnS}$ quantum dots ${ }^{43}$ (QDs) that have a broad two-photon absorption spectrum and high fluorescence quantum efficiency (see Methods, Supplementary Fig. 1 and Supplementary Note 1). Nanopipettes were then slowly advanced towards the spine of interest. When performing targeted spine recordings in slices, layer 5 pyramidal neurons were identified and the procedure described above in cultures was repeated. The nanopipette was subsequently advanced at an oblique angle to guarantee registry (Supplementary Fig. 2).

\section{Nanopipette/cell interface}

Nanopipette entry into the cellular membrane can occur either through impalement or electroporation. In impalement-based entry the nanopipette pierces the cell membrane, similar to conventional sharp microelectrode ${ }^{44}$ operation (model A, Fig. 2a).

Electroporation-induced entry, on the contrary, occurs when the pipette gains intracellular access through an induced nanopore in the lipid bilayer (model B, Fig. 2b). Model A usually results in the formation of a permanent membrane shunt resistance ${ }^{45}$ ( $\left.R_{\text {shunt }}\right)$. The $R_{\text {shunt }}$ in conjunction with the $R C$ time constant of the pipette $\left(R_{\mathrm{e}} C_{\mathrm{e}}\right)$ and the membrane $\left(R_{\mathrm{m}} C_{\mathrm{m}}\right)$ causes a pronounced transient during entry, which plays an important role in determining the steady-state resting membrane potential (RMP) (Supplementary Fig. 3 and Supplementary Note 2). Here $R_{\mathrm{e}}, R_{\mathrm{m}}, C_{\mathrm{e}}$ and $C_{\mathrm{m}}$ denote the pipette and cell membrane resistance and capacitance, respectively. If $R_{\text {shunt }}$ is much larger than $R_{\mathrm{m}}$, the trough in the impalement transient is close to the actual RMP. Transient analysis of the circuit described in model A, clearly suggests that in the presence of an appreciable $R_{\text {shunt }}$, small structures $\left(C_{\mathrm{m}} \sim 0.001-\right.$ $0.1 \mathrm{pF}$ ) dissipate charge quickly, precluding an accurate estimate of the RMP. Model B on the contrary, results in attenuation of the signal due to the resistive divider formed at the pipette/membrane interface. Here, $R_{\text {pore }}, R_{\text {seal }}$ and $C_{\mathrm{m}}$ influence the RMP and overall steady-state condition. If $R_{\text {pore }}$ is extremely high, the shunting effect is negligible (Supplementary Fig. 3) but intracellular voltages are scaled.

Nanoelectrodes exhibit an increased impedance that subsequently leads to filtering effects $^{29,37,44,46}$ (that is, via $R_{\mathrm{e}} C_{\mathrm{e}}$ ). To reduce this filtering, the filling solution comprised of $3 \mathrm{M} \mathrm{KCl}$ to ensure lowest possible resistance. The extracellular chloride concentration of $126 \mathrm{mM}$, however, creates an ionic gradient right at the tip of the pipette, which influences and determines the measured pipette resistance $R_{\mathrm{e}}$, current noise and 3-dB bandwidth ( $f_{3 \mathrm{~dB}}$ ) (see Methods and Supplementary Fig. $5 \mathrm{a}-\mathrm{e}$ ). The analytically determined tip diameter under such conditions was found to be in close agreement with scanning electron microscopy (SEM) measurements (Supplementary Fig. 5c), and provided information on the optimal range of tip diameters to use. To mitigate the effect of pipette wall capacitance, 
active capacitive compensation (Supplementary Fig. 6) was delivered through the amplifier. Together with the measured $R_{\mathrm{e}} \sim 60-240 \mathrm{M} \Omega$, this ensured highest recording bandwidth (see Methods) with $f_{3 \mathrm{~dB}} \sim 1-2 \mathrm{kHz}$. An accurate measurement of $f_{3 \mathrm{~dB}}$ was critical to the deconvolution procedure to correct for electrode filtering effects (see Methods and Supplementary Note 2).

\section{Somatic and somato-dendritic recordings}

We first compared somatic nanopipette recordings of action potentials (APs) in hippocampal cultures against conventional patch and sharp microelectrode recordings (Fig. 3a). The nanopipette was advanced into the soma and a brief oscillation was applied to gain intracellular access. On entry, we observed spontaneous APs and were able to elicit an AP response through brief current injection if required. The characteristics of recorded signals could be well explained by model A (Fig. 2a). We found that AP properties, namely, rise time, decay time, half width and RMP (Fig. 3b) compared favourably to conventional patch and sharp microelectrodes (tip diameter $\sim 100 \mathrm{~nm}$ ). However, RMP obtained using both the nanopipette $(-51 \pm 9.4 \mathrm{mV}, n=5)$ and the sharp microelectrode $(-61 \pm 14 \mathrm{mV}, n=5)$ clearly indicated the presence of a membrane shunt, which resulted in a slightly depolarized RMP (Fig. 3b). Input resistances measured using hyperpolarizing current pulses, ranged from 90 $140 \mathrm{M} \Omega$ for the whole-cell recordings and 78-120 $\mathrm{M} \Omega$ for nanopipette recordings $(n=3)$, suggesting that even in the presence of the shunt, nanopipettes were reporting physiologically relevant values. AP amplitudes and decay times, however, showed that nanopipette recordings were filtered due to the pipette's impedance, the effect of which could be deconvolved (Supplementary Fig. 3 and Supplementary Note 2) to reveal full signal recovery. Somato-dendritic recordings (Fig. $3 \mathrm{c}-\mathrm{e}$ ) confirmed that the nanopipettes were able to faithfully track bAPs (Fig. 3c), EPSPs (Fig. 3b) and inhibitory post-synaptic potentials (IPSPs) in the dendrite without the need for signal averaging. One important difference between somatic and dendritic recordings with nanopipettes is that with dendritic recordings we did not impale the pipette into the dendrite but rather applied a gentle 'touch and buzz' to electroporate the membrane (model B in Figs $2 b$ and 3f, see Methods). When a similar touch-and-buzz protocol was applied to the soma during a simultaneous whole-cell patch-clamp recording, we observed highly attenuated APs in the nanopipette channel (Fig. $3 \mathrm{~g}$ ). The 'buzz' could either be an oscillation or a voltage pulse. However, using model B (Fig. 2b) and the measured pipette properties, we were able to recover the amplitude and timescale of the AP transient (Fig. 3g) through deconvolution (see Methods and Supplementary Note 2). Together, these recordings demonstrate that nanopipettes are capable of resolving both APs and subthreshold activity with high SNR.

Simultaneous somato-dendritic recordings in slices (Fig. 4a,b) revealed stable registration of bAPs (Fig. 4c,d) with high SNR. Both impalement- and electroporation-based nanopipette entry were compared. Although, impalement-based entry resulted in higher SNR, electroporation-based entry enabled more stable and reliable recordings (Fig. 4c, top), which when deconvolved, closely followed the somatic AP (Fig. 4c, bottom). Impalement often caused a resistance drop over time, as observed from the patch-clamp recording, and also resulted in shorter recording times due to the formation of an appreciable membrane shunt (not shown). This indicates that to prolong the recordings in small structures (such as 
dendrites, spines and accompanying dendritic shafts), it is prudent to use electroporationbased entry rather than to physically impale the structure. We corroborated this effect in dendrites via simultaneous nanopipette recordings and $\mathrm{Ca}^{2+}$ imaging (Supplementary Fig. 7). We observed that with weak stimulation (that is, touch and buzz with a short-duration electroporation pulse application), duration of recordings could last longer (max 15-30 min), signals were stable over time (Fig. 4d) and concomitant $\mathrm{Ca}^{2+}$ imaging and electrophysiology was possible. Mechanical advancement followed by strong electroporation (buzz with either longer pulses or increased frequency of stimulation), however, improved the SNR due to the lower pore resistance, but resulted in a concomitant shunt, which was reflected by the rise in baseline fluorescence (Supplementary Fig. 7 and Supplementary Note 3).

Nanopipette recordings in the soma, however, were not affected by the impalement ( $\sim 0 \mathrm{mV}$ amplitudes with half widths $\sim 2 \mathrm{~ms}$; Fig. $4 \mathrm{e}$ ), which further corroborates the transient models (Supplementary Fig. 3) and specifically the effect of membrane capacitance on the recording. Together, somato-dendritic recordings in culture and slice preparations indicate that AP amplitudes and timescales are unchanged right up to the spine neck. Given previously reported impedance estimates of the neck $(\sim 500 \mathrm{M} \Omega)^{10}$ and the spine head $(\sim \text { several giga-ohms at rest })^{47}$, AP invasion into the spine should occur without any loss in signal amplitude, on the basis of electrical equivalent-circuit analyses.

\section{Direct electrical recordings from dendritic spines}

Dendritic spines were located along both the main apical dendrite and proximal oblique branches. Quantum-dot-coated nanopipettes were then steered towards the targeted spine and a touch-and-buzz approach was used to gain intracellular access. The electroporation buzz results in the formation of a small pore in the membrane ${ }^{48}$ (Fig. 5a). This was performed in both culture (Fig. 5b) and slice preparations (Fig. 5c). Since the nanopipette tip was too small to effectively allow dye to diffuse out, pipette entry into the spine was corroborated through QD desorption from the tip surface. The exact mechanism of QD desorption is unclear but we reason that it could occur during the insertion and interaction of the pipette tip with the lipid-bilayer core, which is hydrophobic. As shown in Fig. 5b, after the QD-coated nanopipette touched the spine and an electroporation buzz was applied, QDs were found on the surface of the spine head, (Fig. 5b, middle). The QDs were subsequently found to move with the spine head (Fig. 5d, right), confirming the targeted approach (see Supplementary Fig. 8 for close up). We first probed spines in culture preparations (Fig. 5df) using simultaneous somato-spine electrophysiology. We observed that both evoked and spontaneous bAPs invade spines with the signals in the nanopipette exhibiting high SNR (Fig. 5d; spine, upper trace, and soma, lower trace) without the need for any signal averaging. Depending on the degree of electroporation and penetration, the amplitude and timescale of the deconvolved bAPs that invaded spines were found to be similar to the somatic AP, suggesting full invasion (Supplementary Fig. 8c,d). Interestingly, however, we often found that as the nanopipette was brought in close proximity to the spine head, the nanopipette recorded APs that preceded the initial rise in the post-synaptic EPSP (measured in the soma) by $\sim 2-4 \mathrm{~ms}$ (Fig. 5e). The timescale between the AP peak and the post-synaptic EPSP rise suggests that in these cases the pre-synaptic axon terminal or axon fibre might 
have been accidentally probed prior to spine entry. Given that the size of the axon fibre is extremely small, it is more likely that we probed the pre-synaptic terminal prior to spine entry. The second peak in the post-synaptic EPSP response is clearly indicative of a polysynaptic connection, commonly found in cultures ${ }^{49}$. Although we did not intend to target the pre-synaptic axon terminal, our results suggest that the nanopipette is also capable of intracellularly interfacing to small pre-synaptic structures. To further corroborate pipette spine interaction and entry, we injected current through optimally compensated (bridge) nanopipettes upon touching the spine and measured the resulting voltage changes in the somatic patch pipette and nanopipette (Fig. 5f), respectively. We clearly observed a fast voltage change in the nanopipette with very little capacitive charging, indicative of a small membrane capacitance. An increase in resistance was also observed. The voltage waveform at the soma, however, was clearly filtered, indicative of $R_{\text {neck }}$ and dendritic impedanceinduced signal conditioning. It is important to point out that the voltage waveform in the spine head could be attenuated/shunted due to the nature of the pipette/spine interface, which precludes a clear measurement of the transient. Extending the study to neocortical slice preparations, we clearly observed bAPs invasion in spines (Fig. 5g) and pre-synaptic APs (Fig. 5h) when the nanopipette entered the putative pre-synaptic axon terminal prior to spine entry. Thus, nanopipettes may be used to study the coupling between the pre-and postsynapse at the single-synapse level. The spike triggered average of raw bAPs (Fig. 5i) waveforms clearly revealed that bAP invade spines with nearly no loss in temporal structure (that is, rise time and half width). However, the amplitude of the bAP is scaled due to the nature of the interfacial impedance, which was easily deconvolved (see Methods and Supplementary Note 2). To characterize the extent to which electroporation and mechanical penetration disturb the physiology of the spine, we performed simultaneous electrophysiology and calcium imaging in the spine head (see Supplementary Figs 9 and 10 and Supplementary Note 3). We found that with the touch-and-buzz approach, the pore formed was small enough such that stable $\mathrm{Ca}^{2+}$ imaging in the spine head was still possible concomitantly along with electrophysiology. This was observed both with the nanopipette in the spine and just after retraction of the nanopipette from the spine head. Mechanical penetration followed by electroporation, however, resulted in a large influx of $\mathrm{Ca}^{2+}$ into the spine head. Such an entry allowed measurements of RMPs and improved the SNR, however, at the cost of overall duration (Supplementary Fig. 11).

On nanopipette entry into the spine head, the RMP of the spine head rapidly reached -65 $\mathrm{mV}$ to $-55 \mathrm{mV}$ (Fig. $5 \mathrm{j}$, top, also see Supplementary Note 2). We immediately observed spontaneous EPSPs with amplitudes reaching $\sim 25 \mathrm{mV}$ to $30 \mathrm{mV}$, rise times of $\sim 1-2 \mathrm{~ms}$ and half widths of $\sim 5 \mathrm{~ms}$ (Supplementary Fig. 12 and Supplementary Note 4). Concomitantly monitored somatic EPSPs, however, were negligible ( $\sim 0.5 \mathrm{mV}$, Fig. $5 \mathrm{j}$, bottom) with near baseline fluctuation. Since the RMP was stable over the time course of the recording, the driving force for the EPSPs was likely unaltered and the variability in EPSP amplitudes is most likely due to differences in quantal content.

With electroporation-induced entry, intracellular access to the spine head occurred across a high pore resistance ( $\left.R_{\text {pore }}\right)$ (Fig. 5a). Although this lead to RMPs and amplitudes being scaled by the voltage divider, it provided a more stable recording configuration and improved the duration of recordings in the spine head (Fig. 5k, top, and Supplementary Figs 
11 and 13). As observed in Fig. (5k, top), raw EPSPs in the spine head (marked by star) were still larger than corresponding EPSPs in the soma that were barely detectable above the noise floor $(\sim 0.5 \mathrm{mV})$. Occasionally, we found that somatic EPSPs $(\sim 1-1.5 \mathrm{mV})$ were detectable, and while some of these somatic EPSPs were clearly from other synaptic inputs, a few peaks were time-locked with spine head EPSPs measured with the nanopipette (Fig. $5 \mathrm{k}$, shaded region and Supplementary Fig. 13). We attribute this time-locked somatic signal to simultaneous co-active inputs that originate from other spines, which integrate to form the somatic signal, and not from the recorded spine (Supplementary Fig. 13).

Our spine recordings indicate that EPSP amplitudes in the spine head are large, exhibit a fast rising phase $(\sim<5 \mathrm{~ms})$ but are greatly attenuated, and sometimes even undetectable in the soma. What is the origin of this strong attenuation of EPSPs? At steady state, the magnitude of the spine head EPSP is determined by the voltage divider expressions between $R_{\text {neck }}$, dendritic resistance $\left(R_{\text {dendrite }}\right)$ and synaptic conductance $\left(G_{\text {syn }}\right)$. But the downstream somatic EPSPs are solely determined by the voltage division between $R_{\text {dendrite }}$ and $R_{\text {neck }}$ (Fig. 5a and equations $(1,2))$ :

$$
\begin{gathered}
\mathrm{EPSP}_{\text {spine_head }}=E_{\text {syn }} \frac{R_{\text {neck }}+R_{\text {dendrite }}}{R_{\text {neck }}+R_{\text {dendrite }}+\frac{1}{G_{\text {syn }}}} \\
\mathrm{EPSP}_{\text {soma/dendrite }}=\mathrm{EPSP}_{\text {spine_head }} \frac{R_{\text {dendrite }}}{R_{\text {neck }}+R_{\text {dendrite }}}
\end{gathered}
$$

where $E_{\mathrm{syn}}$ is the synaptic reversal potential. Thus, the EPSP attenuation depends on both $R_{\text {neck }}$ and $R_{\text {dendrite. }}$. Since the spines studied were very close to the soma, and the $R_{\text {dendrite }}$ of pyramidal cells ranges $\sim 10-15 \mathrm{M} \Omega$ within $100 \mu \mathrm{m}$ from the soma ${ }^{10}$, our measured spine head EPSP amplitudes of $\sim 25 \mathrm{mV}$ and somatic EPSPs of $\sim 0.5-1 \mathrm{mV}$ would imply a high $R_{\text {neck }}$. This would suggest that EPSPs are isolated from the parent dendrite by the spine neck. To estimate $R_{\text {neck }}$, EPSP amplitudes and RMPs, we used equivalent-circuits analysis and measured time constants (Fig. 51, Methods and Supplementary Note 2), and found a range for $R_{\text {neck }}(425 \pm 101.62 \mathrm{M} \Omega, n=6$ spines, Fig. 5l, left), EPSP amplitudes (26.70 $\pm 13.8 \mathrm{mV}$, total of 39 EPSPs, $n=6$ spines, Fig. 51 , middle), and RMPs $(-52 \pm 6.38 \mathrm{mV}, n=$ 3 spines, Fig. 51, right) in the spine head. These estimates are consistent with earlier theoretical and optical reports ${ }^{2,10}$. However, since our selection of spines had relatively long necks $(\sim 1 \mu \mathrm{m})$, and that EPSP attenuation likely depends on neck length ${ }^{15}$, our estimates of $R_{\text {neck }}$ may be higher than previous reports. Lastly, EPSPs and the observed RMPs (Fig. 5j) in the spine head during spontaneous entry were in close agreement with the deconvolved parameters (Fig. 5l, middle and right). 


\section{Conclusion}

We have demonstrated a direct intracellular interface to spines using nanopipettes and detected bAPs and EPSPs in the spine head without the need for signal averaging. We find that spine head EPSPs are large $(\sim 26 \mathrm{mV})$ compared to downstream somatic potentials $(\sim 0.5$ $\mathrm{mV}$ ), and, using circuit models and experimentally determined parameters, we estimate a high neck resistance. Our results thus suggest that EPSP attenuation from the spine to soma is influenced by spine neck resistance, which could play an important role in shaping synaptic transmission and enabling nonlinear dendritic signal processing ${ }^{1,10}$.

Additional work will make this technique even more precise and accurate. For example, the use of lipid layers and biomimetic coatings ${ }^{50-52}$ could improve the seal resistance, enabling stable long-term recordings in spines and simultaneous spine and dendritic recordings to directly measure $R_{\text {neck}}$. The demonstration of nanopipettes as direct electrical interfaces to spines and putative pre-synaptic axon terminals presented in this study will inform on future designs and expand the scope of nanoelectrodes in the electrophysiology 53 of small neuronal structures.

\section{Methods}

\section{Primary hippocampal neuronal cultures and acute slice preparation}

Animal handling and experimentation was done according to the US National Institutes of Health and Columbia Institutional Animal Care and Use Committee guidelines.

Hippocampal neuronal cultures were generated from embryonic day (E)18 or postnatal day (P)0 neonatal C57BL/6 mice as previously described ${ }^{54}$, with few modifications. Briefly, pregnant dams or neonatal pups were anesthetized and euthanized. Hippocampi were dissected and subsequently incubated in $0.25 \%$ Trypsin + EDTA (Life Technologies, 25200 056) and $5 \mu \mathrm{g} \mathrm{ml}^{-1}$ DNAse I (Sigma, D5025) at $37^{\circ} \mathrm{C}$ for $20 \mathrm{~min}$ with gentle agitation. After two washes with plating medium containing $5 \mu \mathrm{g} \mathrm{m}{ }^{-1}$ DNase I, hippocampi were gently triturated using fire-polished glass Pasteur pipettes until a homogenous cell suspension was obtained. Cells were then plated onto $12 \mathrm{~mm}$ poly-1-lysine coated coverslips (Corning, 354085), with and without an astroglial feeder layer. Astroglial feeder layers were produced by exposing previously plated days in vitro (DIV) 4-7 hippocampal primary cultures to a high-glutamate excito-toxic solution for $15 \mathrm{~min}$, allowing a minimum of two additional days for nonviable neurons to detach from the coverslip surface. Once the astroglial layer reached desired confluency, $5 \mu \mathrm{M}$ cytosine $\beta$-D-arabinofuranoside (SigmaAldrich, C1768) was added to culture medium to inhibit further proliferation. Astroglial feeder layers were ready for use approximately 1 week after plating. Neurons were cultured for 2-4 weeks prior to experimentation, with maximal spine density occurring between 14 28 DIV. Cultures were maintained at $37{ }^{\circ} \mathrm{C}$ under $5 \% \mathrm{CO}_{2} .50 \%$ of culture medium was exchanged with fresh medium every 3 days.

For acute slice experiments, coronal sections of the neocortex of P7 to P20 old C57BL/6 mice of both sexes were prepared using a Leica VT1200S vibratome. The animal was decapitated (following deep anesthesia via inhalation of isoflurane in case of animals older than P12), and the brain quickly removed. Slices of $300 \mu \mathrm{m}$ thickness were prepared in ice- 
cold slicing solution containing (in $\mathrm{mM}$ ): $93 \mathrm{~N}$-methyl-D-glucamine, $2.5 \mathrm{KCl}, 1.2 \mathrm{NaH}_{2} \mathrm{PO}_{4}$, $30 \mathrm{NaHCO}_{3}, 20 \mathrm{HEPES}, 25$ glucose, $5 \mathrm{Na}$-ascorbate, $3 \mathrm{Na}$-pyruvate, $10 \mathrm{MgSO}_{4}, 0.5 \mathrm{CaCl}_{2}$, $\mathrm{pH}$ adjusted with $\mathrm{HCl}$ to 7.3 , bubbled with $95 \% \mathrm{O}_{2}$ and $5 \% \mathrm{CO}_{2}$. After a short recovery period (4-8 $\mathrm{min}$ ) in $35-37^{\circ} \mathrm{C}$ warm slicing solution, slices were kept at room temperature in artificial cerebral spinal fluid (ACSF) until transferred into a recording chamber.

\section{QD preparation}

Refer to Supplementary Note 1 for detailed preparation.

\section{Electrophysiology}

Neurons (culture and slices) were visualized using an Olympus BX-61 or BX50WI microscope equipped with oblique illumination and a water immersion $40 \times / 0.8$ numerical aperture (NA) objective (Olympus). Whole-cell patch-clamp recordings (pipette resistance 4-7 $\mathrm{M} \Omega$ ) were obtained using pipettes pulled from borosilicate glass $(1.5 \mathrm{~mm}$ and $1 \mathrm{~mm}$ outer diameter (OD), $0.86 \mathrm{~mm}$ and $0.5 \mathrm{~mm}$ inner diameter (ID), Sutter Instruments) and established using a Multiclamp 700B amplifier (Molecular Devices). The RMP was maintained at $-65 \mathrm{mV}$ to $-70 \mathrm{mV}$ via slow current injection if necessary. The external bath contained ACSF comprising (in mM): $126 \mathrm{NaCl}, 26 \mathrm{NaHCO}_{3}, 1.145 \mathrm{NaH}_{2} \mathrm{PO}_{4}, 10$ glucose, $3 \mathrm{KCl}, 2 \mathrm{MgSO}_{4}$ and $2 \mathrm{CaCl}_{2}$, osmolarity $\sim 300 \mathrm{mOsm}$. Patch pipettes were filled with internal solution containing (in mM): $130 \mathrm{~K}$-gluconate, $5 \mathrm{NaCl}, 2 \mathrm{MgSO}_{4}, 10 \mathrm{HEPES}, 5$ EGTA, 4 MgATP, $0.4 \mathrm{Na}_{2} \mathrm{GTP}, 7 \mathrm{Na}_{2}$-phospocreatine, 2 pyruvic acid, 0.002-0.01 Alexa 488, $\mathrm{pH}$ adjusted to 7.2, 280-290 mOsm). For $\mathrm{Ca}^{2+}$ imaging experiments, 50-100 $\mu \mathrm{M}$ Fluo4 and 1-5 $\mu \mathrm{M}$ Alexa 594 were added to the internal solution. Nanopipettes (inner tip diameters $\sim 15-30 \mathrm{~nm}$ ) were fabricated using a P-2000 laser puller (Sutter Instruments) and made from quartz capillaries ( $1 \mathrm{~mm}$ OD, $0.5 \mathrm{~mm}$ ID, Sutter Instruments). Program parameter range: Heat: 770-850; FIL: 5; VEL: 45-52; DEL: 130-155; PUL: 155-180. It is important to note that program parameters vary from puller to puller. The nanopipettes were first back-filled and then front-filled with $3 \mathrm{M} \mathrm{KCl}$ (resistance range $60-240 \mathrm{M} \Omega$ ), dipped in QD solution (3-5 times rapidly to ensure uniform coating) and carefully bridge compensated prior to recording through the built-in module in the multiclamp 700B and Axon 900A amplifiers (Molecular Devices). The Axon 900A was specifically used with pipette impedances above $180 \mathrm{M} \Omega$ due to the improved input impedance (boot strapped, $R_{\text {in }}$ $>1,000 \mathrm{G} \Omega$ ) and capacitance compensation range of the amplifier. Bandwidth extraction was carried out both before and after the application of compensation through the bridge mode. Briefly, a current pulse was injected. The resulting voltage response at the rising edge of the current pulse (Fig. $2 \mathrm{~g}$ ) was fit to extract the time constant $\tau_{\mathrm{P}}$. Dividing $\tau_{\mathrm{P}}$ by the measured value of the pipette resistance $R_{\mathrm{e}}$, the pipette wall capacitance $C_{\mathrm{e}}$ was determined. The $3-\mathrm{dB}$ bandwidth was calculated using the expression $f_{3 \mathrm{~dB}}=1 /\left(2 \pi R_{\mathrm{e}} C_{\mathrm{e}}\right)$. The 3-dB bandwidth was also corroborated using impedance spectroscopy ( $\mathrm{CH}$ Instruments 760D). Entry into spines was carried out either through mechanical impalement or electroporation ('touch and buzz'). For electroporation-induced entry, brief $1 \mathrm{~V}, 500 \mu \mathrm{s}$ pulses were applied until entry was established. Alternatively, oscillations of varying durations (50-500 $\mu$ s) were utilized until entry was established. Offsets ( -2 to $-5 \mathrm{mV}$ ) were zeroed prior to spine entry. Current clamp signals from both the nanopipette and patch pipette were filtered by a 4-pole Bessel filter (4 $\mathrm{kHz}$ ) and acquired at $10 \mathrm{kHz}$ (NI-DAQ 6251, National Instruments) using a PC equipped 
with custom software $\left(\right.$ PackIO $\left.^{55}\right)$ written in LabView (National Instruments). Nanopipette inner diameters (I.D.) were calculated through the expression I.D. $=2 /\left(\pi R_{P} \sigma \tan (\theta / 2)\right)$, where $R_{\mathrm{p}}$ is the measured pipette resistance, $\theta$ is the measured cone angle and $\sigma$ is the overall conductivity. Tip diameters were measured by first coating the nanopipettes with $\sim 10$ $\mathrm{nm}$ of gold with a sputter coater (Cressington 108 Sputter Coater), then imaged with a scanning electron microscope (SEM, Hitachi S-4700, $n=5$ ). The beam current was set to 10 $\mu \mathrm{A}$ and the acceleration voltage was $15 \mathrm{keV}$. The cone angle was extracted using ImageJ (US, National Institutes of Health) (range $~ 2.5-3.0^{\circ}$ ). Resistances were first measured when both the internal and external bath solutions were matched $(3 \mathrm{M} \mathrm{KCl})$ to avoid rectification. The assumed $\sigma \sim 30 \mathrm{~S} \mathrm{~m}^{-1}$ yielded a near perfect fit to the SEM measured tip diameters 25$30 \mathrm{~nm}$. With the external bath replaced with ACSF and with the same pipette filling solution ( $3 \mathrm{M} \mathrm{KCl}$ ), $\sigma$ was found to reduce $\sim 11 \mathrm{~S} \mathrm{~m}^{-1}$ to match the measured resistance with measured tip diameters indicating a large concentration gradient at the tip. These results were corroborated with $1 \mathrm{M} \mathrm{KCl}, \sigma \sim 11 \mathrm{~S} \mathrm{~m}^{-1}$ (ref. 56) as the filling solution and was found to be in agreement with the measured tip diameters.

\section{Deconvolving EPSP amplitudes, RMP and the $\boldsymbol{R}_{\text {neck }}$}

When bAPs invade spines, they encounter a voltage divider ${ }^{57}$, that is, across $R_{\text {neck }}$ and $R_{\mathrm{m}}$ (Fig. 5a). Here, $R_{\mathrm{m}}$ denotes the passive membrane resistance and is much larger than $R_{\text {neck }}$. This voltage divider facilitates full bAP invasion. To register full bAP amplitudes in the spine head using nanopipettes, $R_{\mathrm{m}}$ needs to be nearly unchanged even after pipette entry. This can occur only if the seal resistance between the pipette and the membrane is extremely high $\left(R_{\text {seal }} \gg R_{\mathrm{m}}\right)$. But what happens if $R_{\text {pore }}$ and $R_{\text {seal }}$ are significantly lower than $R_{\mathrm{m}}$ ? $R_{\text {pore }}$ together with $R_{\text {seal }}$ not only forms a voltage divider at the spine head nanopipette interface (Fig. 5a) but also lowers $R_{\mathrm{m}}$. This causes a lowering in the bAP amplitude that invades the spine from the dendrite. Since this attenuation occurs across the neck resistance and the pipette/spine head interface, an accurate measure of the pipette interface properties should shed light on the possible range of $R_{\text {neck }}$. By estimating the voltage divider ratio at the spine head/nanopipette interface (Fig. 5a) from RMP measurements and voltage transient on spine head entry, estimating the measured bAP amplitudes in the spine head, extracting a range of $R_{\text {seal }}$ and $R_{\text {pore }}$ values from simultaneous patch-nanopipette recordings in the soma under identical entry conditions, and by inverse-filtering the waveforms using the measured electrode time constants (that is, $R_{\mathrm{e}} C_{\mathrm{e}}$ ), we calculated the range for $R_{\text {neck }}$, EPSP amplitudes and RMPs (Fig. 51 and see Supplementary Note 2 for details).

\section{Imaging}

Two-photon laser scanning microscopy of spines ${ }^{58}$ ensures minimal photodamage due to the short dwell time per pixel, allows for prolonged experimentation and guarantees rejection of background fluorescence due to excitation in a small volume. Targeted nanopipette experiments were performed with a custom-made two-photon laser scanning microscope based on a modified Olympus BX50WI microscope (Olympus), controlled with either Fluoview (Olympus) or Scan Image 2015 (Vidrio Technologies), with a Ti:sapphire laser at 800 or $940 \mathrm{~nm}$ as light source (Coherent) model: Chameleon Ultra II, $140 \mathrm{fs}$ pulses, $80 \mathrm{MHz}$ repetition rate. A $40 \times / 0.8 \mathrm{NA}$ objective (Olympus) was used to acquire images. Images were collected using Fluoview 2.1.22 (Olympus) or Scan Image 2015a (Vidrio Technologies). 
Laser power was modulated by a Pockels cell (350-160, Conoptics). Optical signals were separated through a 565 long-pass dichroic, using a 535/20 emission filter for the green signal and a 605/20 emission filter for the red signal, and amplified through photomultiplier tubes (H7422-P40 Hamamatsu) connected to a signal preamplifier (Model 5113, Signal Recovery AMETEK Advanced Measurement Technology). The fluorescent fill of the patched neuron and nanopipette QD coating was confirmed before two-photon imaging using epifluorescent illumination via a mercury lamp and appropriate emission filters.

For two-photon $\mathrm{Ca}^{2+}$ imaging, changes in fluorescence were recorded using the green channel (535/20 emission filter), via Scan Image control in bidirectional scanning mode with an acquisition rate of $15 \mathrm{~Hz}$. Neuronal morphology and spines were located using the red channel (605/20 emission filter). Excitation wavelength was $800 \mathrm{~nm}$. Motion correction was used to account for movement during experimentation ${ }^{59}$. Signals were extracted using custom-written Matlab software. $\Delta f f f$ was calculated by defining a baseline fluorescence $f_{0}$ (100 frames) and normalizing the signal trace $f$ to this value. Spine fluorescence signals were extracted using a hand-drawn region of interest around the spine. Care was taken not to include the dendrite. Background fluorescence was found to be extremely low and was not subtracted.

\section{Supplementary Material}

Refer to Web version on PubMed Central for supplementary material.

\section{Acknowledgments}

K.J. would like to thank M. Barbic of the applied physics and instrumentation group at Janelia Farms for initial assistance with initial SEM imaging. This work was supported by the National Institute of Mental Health (NIMH) (R01MH101218, R01MH100561) and the Kavli Institute of Brain Science. This material is also based on work supported by, or in part by, the US Army Research Laboratory and the US Army Research Office under contract number W911NF-12-1-0594 (MURI).

\section{References}

1. Yuste R. Electrical compartmentalization in dendritic spines. Annu. Rev. Neurosci. 2013; 36:429449. [PubMed: 23724997]

2. Grunditz $\AA$, Holbro N, Tian L, Zuo Y, Oertner TG. Spine neck plasticity controls postsynaptic calcium signals through electrical compartmentalization. J. Neurosci. 2008; 28:13457-13466. [PubMed: 19074019]

3. Hao J, Oertner TG. Depolarization gates spine calcium transients and spike-timing-dependent potentiation. Curr. Opin. Neurobiol. 2012; 22:509-515. [PubMed: 22051693]

4. Grutzendler J, Kasthuri N, Gan W-B. Long-term dendritic spine stability in the adult cortex. Nature. 2002; 420:812-816. [PubMed: 12490949]

5. Yang G, Pan F, Gan W-B. Stably maintained dendritic spines are associated with lifelong memories. Nature. 2009; 462:920-924. [PubMed: 19946265]

6. Yuste R, Bonhoeffer T. Morphological changes in dendritic spines associated with long-term synaptic plasticity. Annu. Rev. Neurosci. 2001; 24:1071-1089. [PubMed: 11520928]

7. Yuste R, Denk W. Dendritic spines as basic functional units of neuronal integration. Nature. 1995; 375:682-684. [PubMed: 7791901]

8. Müller W, Connor JA. Dendritic spines as individual neuronal compartments for synaptic $\mathrm{Ca}^{2+}$ responses. Nature. 1991; 354:73-76. [PubMed: 1682815] 
9. Bloodgood BL, Sabatini BL. Neuronal activity regulates diffusion across the neck of dendritic spines. Science. 2005; 310:866-869. [PubMed: 16272125]

10. Harnett MT, Makara JK, Spruston N, Kath WL, Magee JC. Synaptic amplification by dendritic spines enhances input cooperativity. Nature. 2012; 491:599-602. [PubMed: 23103868]

11. Perkel DH, Perkel DJ. Dendritic spines: role of active membrane in modulating synaptic efficacy. Brain Res. 1985; 325:331-335. [PubMed: 2579708]

12. Shepherd G, et al. Signal enhancement in distal cortical dendrites by means of interactions between active dendritic spines. Proc. Natl Acad. Sci. USA. 1985; 82:2192-2195. [PubMed: 3856892]

13. Miller JP, Rall W, Rinzel J. Synaptic amplification by active membrane in dendritic spines. Brain Res. 1985; 325:325-330. [PubMed: 2983830]

14. Bloodgood BL, Giessel AJ, Sabatini BL. Biphasic synaptic Ca influx arising from compartmentalized electrical signals in dendritic spines. PLoS Biol. 2009; 7:1-10.

15. Araya R, Jiang J, Eisenthal KB, Yuste R. The spine neck filters membrane potentials. Proc. Natl Acad. Sci. USA. 2006; 103:17961-17966. [PubMed: 17093040]

16. Acker CD, Yan P, Loew LM. Single-voxel recording of voltage transients in dendritic spines. Biophys. J. 2011; 101:L11-L13. [PubMed: 21767473]

17. Segev I, Rall W. Computational study of an excitable dendritic spine. J. Neurophysiol. 1988; 60:499-523. [PubMed: 2459320]

18. Koch C, Zador A. The function of dendritic spines: devices subserving biochemical rather than electrical computation. J. Neurosci. 1993; 13:413-422. [PubMed: 8426220]

19. Svoboda K, Tank DW, Denk W. Direct measurement of coupling between dendritic spines and shafts. Science. 1996; 272:716-719. [PubMed: 8614831]

20. Tønnesen J, Katona G, Rózsa B, Nägerl UV. Spine neck plasticity regulates compartmentalization of synapses. Nat. Neurosci. 2014; 17:678-685. [PubMed: 24657968]

21. Palmer LM, Stuart GJ. Membrane potential changes in dendritic spines during action potentials and synaptic input. J. Neurosci. 2009; 29:6897-6903. [PubMed: 19474316]

22. Hochbaum DR, et al. All-optical electrophysiology in mammalian neurons using engineered microbial rhodopsins. Nat. Methods. 2014; 11:825-833. [PubMed: 24952910]

23. Acker CD, Hoyos E, Loew LM. EPSPs measured in proximal dendritic spines of cortical pyramidal neurons. eneuro. 2016; 3:1-13.

24. Popovic MA, Gao X, Carnevale NT, Zecevic D. Cortical dendritic spine heads are not electrically isolated by the spine neck from membrane potential signals in parent dendrites. Cerebral Cortex. 2012; 24:385-395. [PubMed: 23054810]

25. Popovic MA, Carnevale N, Rozsa B, Zecevic D. Electrical behaviour of dendritic spines as revealed by voltage imaging. Nat. Commun. 2015; 6:8436. [PubMed: 26436431]

26. Nuriya M, Jiang J, Nemet B, Eisenthal KB, Yuste R. Imaging membrane potential in dendritic spines. Proc. Natl Acad. Sci. USA. 2006; 103:786-790. [PubMed: 16407122]

27. Qing Q, et al. Free-standing kinked nanowire transistor probes for targeted intracellular recording in three dimensions. Nat. Nanotech. 2014; 9:142-147.

28. Qing Q, et al. Nanowire transistor arrays for mapping neural circuits in acute brain slices. Proc. Natl Acad. Sci. USA. 2010; 107:1882-1887. [PubMed: 20133836]

29. Robinson JT, et al. Vertical nanowire electrode arrays as a scalable platform for intracellular interfacing to neuronal circuits. Nat. Nanotech. 2012; 7:180-184.

30. Tian B, et al. Three-dimensional, flexible nanoscale field-effect transistors as localized bioprobes. Science. 2010; 329:830-834. [PubMed: 20705858]

31. Xie C, Lin Z, Hanson L, Cui Y, Cui B. Intracellular recording of action potentials by nanopillar electroporation. Nat. Nanotech. 2012; 7:185-190.

32. Singhal R, et al. Multifunctional carbon-nanotube cellular endoscopes. Nat. Nanotech. 2011; 6:5764.

33. Novak P, et al. Nanoscale-targeted patch-clamp recordings of functional presynaptic ion channels. Neuron. 2013; 79:1067-1077. [PubMed: 24050398]

34. Spira ME, Hai A. Multi-electrode array technologies for neuroscience and cardiology. Nat. Nanotech. 2013; 8:83-94. 
35. Fendyur A, Spira ME. Toward on-chip, in-cell recordings from cultured cardiomyocytes by arrays of gold mushroom-shaped microelectrodes. Front. Neuroeng. 2012; 5:1-10. [PubMed: 22347181]

36. Hai A, Shappir J, Spira ME. In-cell recordings by extracellular microelectrodes. Nat. Methods. 2010; 7:200-202. [PubMed: 20118930]

37. Angle MR, Schaefer AT. Neuronal recordings with solid-conductor intracellular nanoelectrodes (SCINEs). PLoS ONE. 2012; 7:e43194. [PubMed: 22905231]

38. Alivisatos AP, et al. Nanotools for neuroscience and brain activity mapping. ACS Nano. 2013; 7:1850-1866. [PubMed: 23514423]

39. $\mathrm{Hu} \mathrm{H}$, Jonas P. A supercritical density of $\mathrm{Na}^{+}$channels ensures fast signaling in GABAergic interneuron axons. Nat. Neurosci. 2014; 17:686-693. [PubMed: 24657965]

40. Stuart GJ, Sakmann B. Active propagation of somatic action potentials into neocortical pyramidal cell dendrites. Nature. 1994; 367:69-72. [PubMed: 8107777]

41. Stuart GJ, Häusser M. Dendritic coincidence detection of EPSPs and action potentials. Nat. Neurosci. 2001; 4:63-71. [PubMed: 11135646]

42. Holthoff K, Zecevic D, Konnerth A. Rapid time course of action potentials in spines and remote dendrites of mouse visual cortex neurons. J. Physiol. 2010; 588:1085-1096. [PubMed: 20156851]

43. Andrasfalvy BK, et al. Quantum dot-based multiphoton fluorescent pipettes for targeted neuronal electrophysiology. Nat. Methods. 2014; 11:1237-1241. [PubMed: 25326662]

44. Purves, R. Microelectrode Methods for Intracellular Recording and Ionophoresis. Academic; 1981.

45. Ince $\mathrm{C}$, et al. Intracellular microelectrode measurements in small cells evaluated with the patch clamp technique. Biophys. J. 1986; 50:1203-1209. [PubMed: 3801578]

46. Brette R, et al. High-resolution intracellular recordings using a real-time computational model of the electrode. Neuron. 2008; 59:379-391. [PubMed: 18701064]

47. Tsay D, Yuste R. On the electrical function of dendritic spines. Trend. Neurosci. 2004; 27:77-83. [PubMed: 15102486]

48. Lin ZC, Xie C, Osakada Y, Cui Y, Cui B. Iridium oxide nanotube electrodes for sensitive and prolonged intracellular measurement of action potentials. Nat. Commun. 2014; 5:3206. [PubMed: 24487777]

49. Bi, G-q, Poo, M-m. Distributed synaptic modification in neural networks induced by patterned stimulation. Nature. 1999; 401:792-796. [PubMed: 10548104]

50. Duan X, et al. Intracellular recordings of action potentials by an extracellular nanoscale field-effect transistor. Nat. Nanotech. 2012; 7:174-179.

51. Fu T-M, et al. Sub-10-nm intracellular bioelectronic probes from nanowire- nanotube heterostructures. Proc. Natl Acad. Sci. USA. 2014; 111:1259-1264. [PubMed: 24474745]

52. Almquist BD, Melosh NA. Fusion of biomimetic stealth probes into lipid bilayer cores. Proc. Natl Acad. Sci. USA. 2010; 107:5815-5820. [PubMed: 20212151]

53. Angle MR, Cui B, Melosh NA. Nanotechnology and neurophysiology. Curr. Opin. Neurobiol. 2015; 32:132-140. [PubMed: 25889532]

54. Beaudoin GM III, et al. Culturing pyramidal neurons from the early postnatal mouse hippocampus and cortex. Nat. Protoc. 2012; 7:1741-1754. [PubMed: 22936216]

55. Packer, AM. Understanding the Nervous System as an Information Processing Machine: Dense, Nonspecific, Canonical Microcircuit Architecture of Inhibition in Neocortex and a Neural Circuit for Angular Velocity Computation PhD thesis. Columbia Univ; 2011.

56. Garaj S, et al. Graphene as a subnanometre trans-electrode membrane. Nature. 2010; 467:190-193. [PubMed: 20720538]

57. Araya R. Input transformation by dendritic spines of pyramidal neurons. Front. Neuroanatomy. 2014; 8:1-18.

58. Denk W, Yuste R, Svoboda K, Tank DW. Imaging calcium dynamics in dendritic spines. Curr. Opin. Neurobiol. 1996; 6:372-378. [PubMed: 8794079]

59. Thevenaz P, Ruttimann UE, Unser M. A pyramid approach to registration based on intensity. IEEE Trans. Image Processing. 1998; 7:27-41. 
a

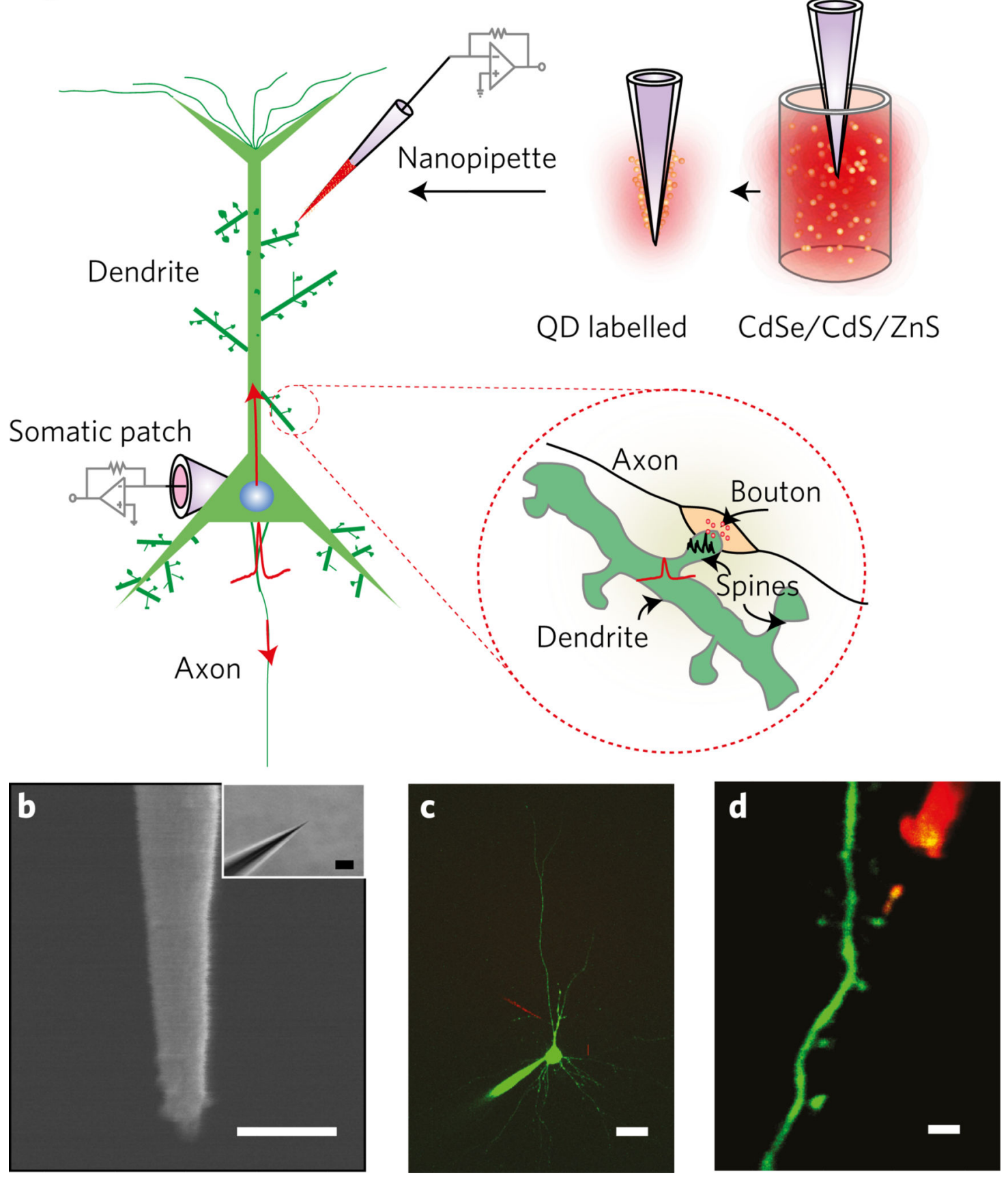

Figure 1. Experimental overview

a, Schematic describing the dual patch-nanopipette recording and associated signal transmission mechanisms to and from the spine. APs originate in the axon hillock and propagate both forwards (towards the post-synaptic cell) and backwards into the dendrites and spines. bAPs invade spines (inset) across the spine neck. Neurotransmitters released from the pre-synaptic axonal terminals activate receptors located on the spine head and cause spine head EPSPs (inset). Nanopipettes are labelled with QDs through adsorption (top right). b, SEM of a typical quartz nanopipette (scale bar, $100 \mathrm{~nm}$ ) and respective bright-field 
image (inset, scale bar, $2 \mu \mathrm{m}$ ). c, Simultaneous somato-dendritic recordings from layer-5 pyramidal cells using QD-coated nanopipettes. Scale bar, $20 \mu \mathrm{m}$. d, Nanopipette probing a spine. Scale bar, $1.5 \mu \mathrm{m}$. 
a

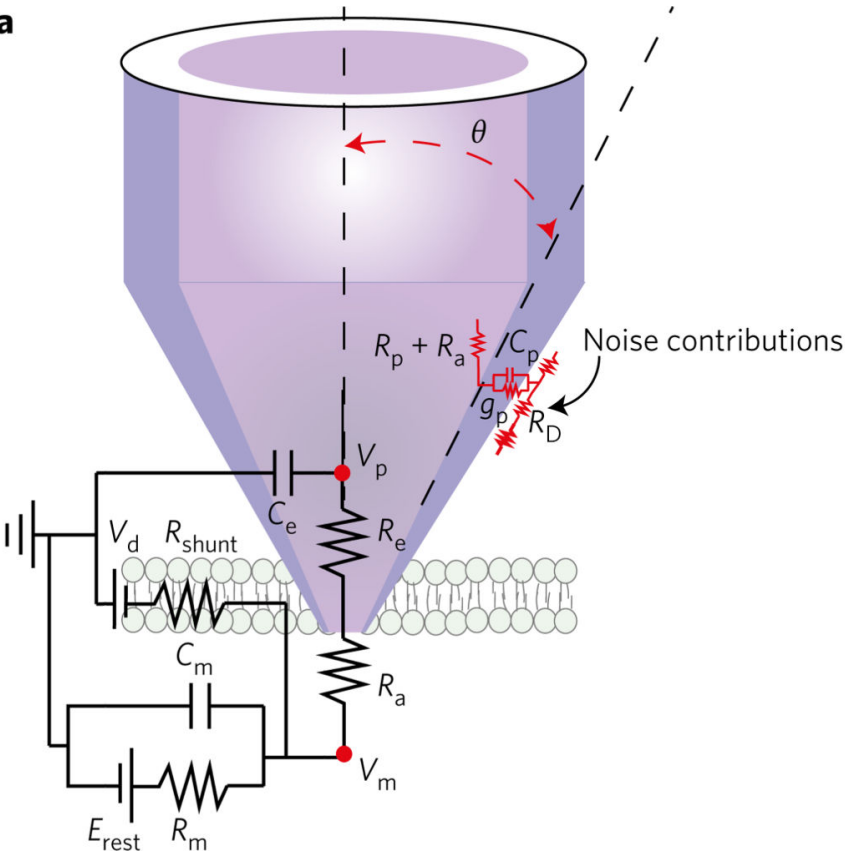

b

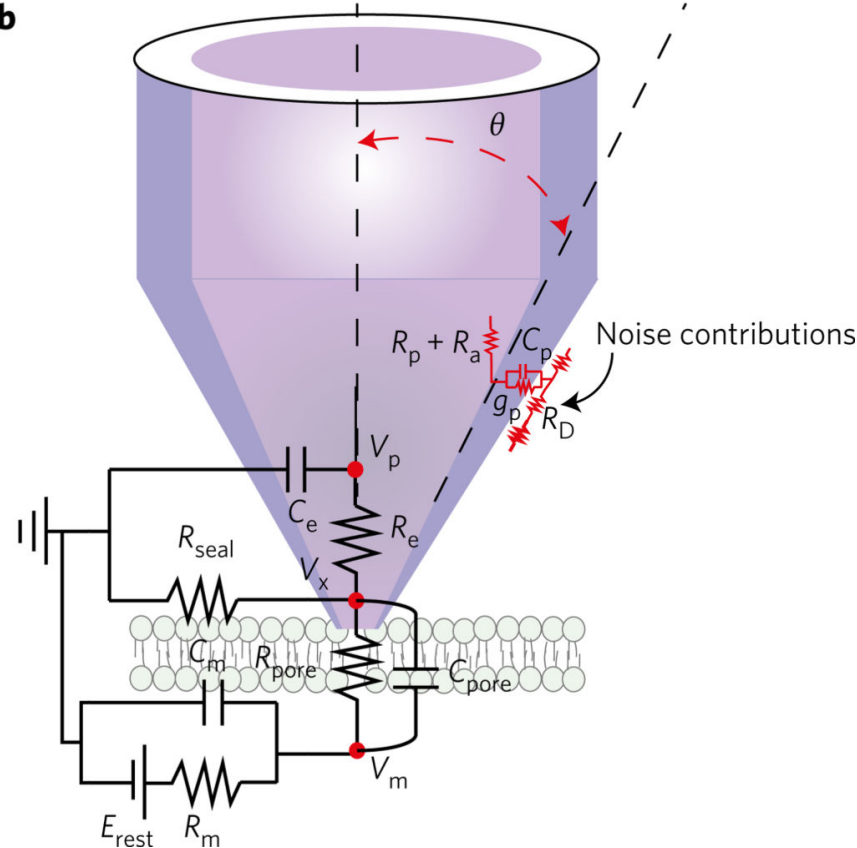

Figure 2. The nanopipette membrane interface a,b, Equivalent circuit describing the nanopipette membrane interface during penetration(a) and electroporation-based (b) entry. Here $R_{\mathrm{e}}$ and $C_{\mathrm{e}}$ denote the pipette resistance and capacitance, respectively. $R_{\mathrm{a}}, R_{\text {shunt }}, R_{\text {seal }}$ and $R_{\text {pore }}$ represent the access, shunt, seal and pore resistance respectively. $R_{\mathrm{m}}$ represents the membrane resistance, $C_{\mathrm{m}}$ the membrane capacitance, $C_{\text {pore }}$ the pore capacitance and $E_{\text {rest }}$ the reversal potential of the leak channels, respectively. All of which combine to determine the RMP. $V_{\mathrm{d}}$ is the diffusion potential, which under grounded bath conditions can be approximated to zero. $\theta$ denotes the inner cone angle, which is used to calculate the tip diameter as a function of resistance. Potential noise contributions are highlighted in red. The parasitic capacitances from the pipette holder and amplifier electronics were found to be negligible and hence omitted for simplification. 

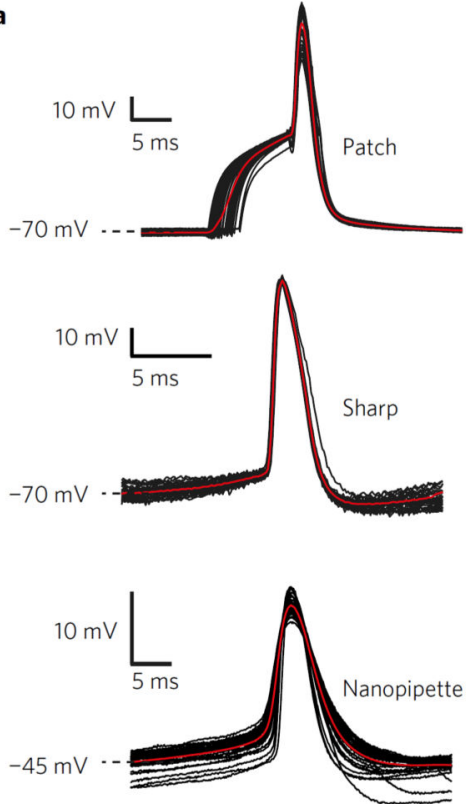

Soma

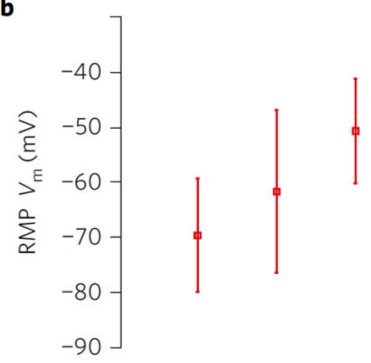

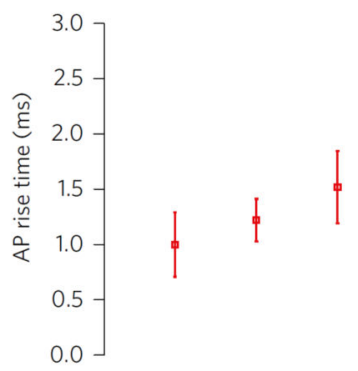
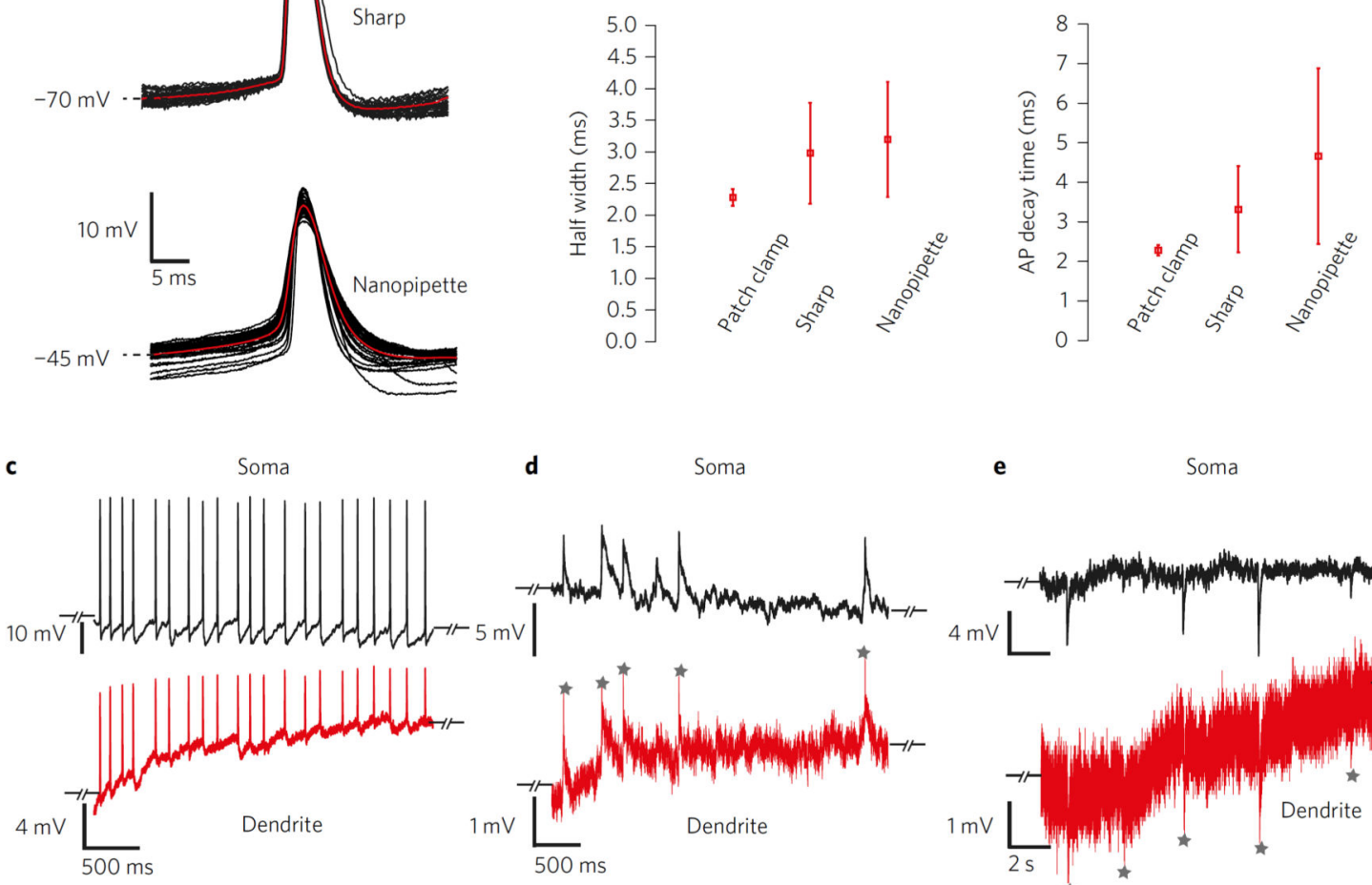

d

Soma

e

Soma

$\mathbf{f}$

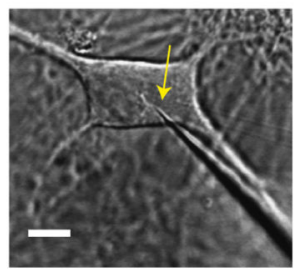

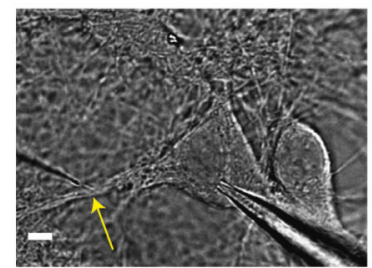

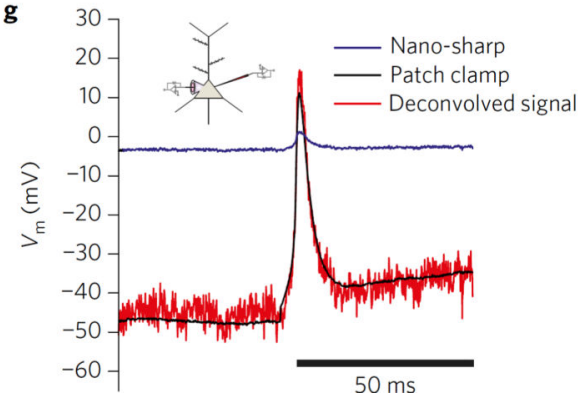

Figure 3. Nanopipette recordings from cultures

SNR and somato-dendritic coupling. a, Spike triggered average (STA) of APs recorded from the soma using patch-clamp (top), $100 \mathrm{~nm}$ glass microelectrodes (middle) and quartz nanopipette electrodes (bottom) under membrane penetration. Note that the initial rise in the patch-clamp recording is due to current injection from the patch electrode. The sharp and nanopipette AP recordings were spontaneous. b, Comparison of RMP, AP rise time, halfwidth and decay time properties between patch, sharp and nanopipette electrodes. Note that nanopipette recordings often result in a membrane shunt leading to a more positive RMP. c- 
e, Somato-dendritic recordings using patch-nanopipette electrodes show simultaneous registry of APs (c), EPSPs (d) and IPSPs (e) in both channels with high SNR. Dendritic recordings were initiated through membrane electroporation. The slow increase in RMP baseline in $\mathbf{c}$ is indicative of the electropore resealing, which causes a gradual change in $R_{\text {pore }}$ Grey stars in d,e denote EPSP and IPSP peaks that can be clearly resolved across the nano-orifice. f, Infrared differential contrast interference (IR-DIC) images of nanopipette electroporation in the somatic membrane (left, scale bar, $5 \mu \mathrm{m}$ ) and dendritic membrane (right, scale bar, $5 \mu \mathrm{m}$ ). $\mathbf{g}$, Deconvolved AP amplitude (through inverse filtering) using measured time constants and voltage divider ratios during a simultaneous patch-nanopipette recording from the same soma. The deconvolved waveform is temporally similar to the actual AP waveform. The nanopipette recording was established through electroporation and not membrane penetration. 

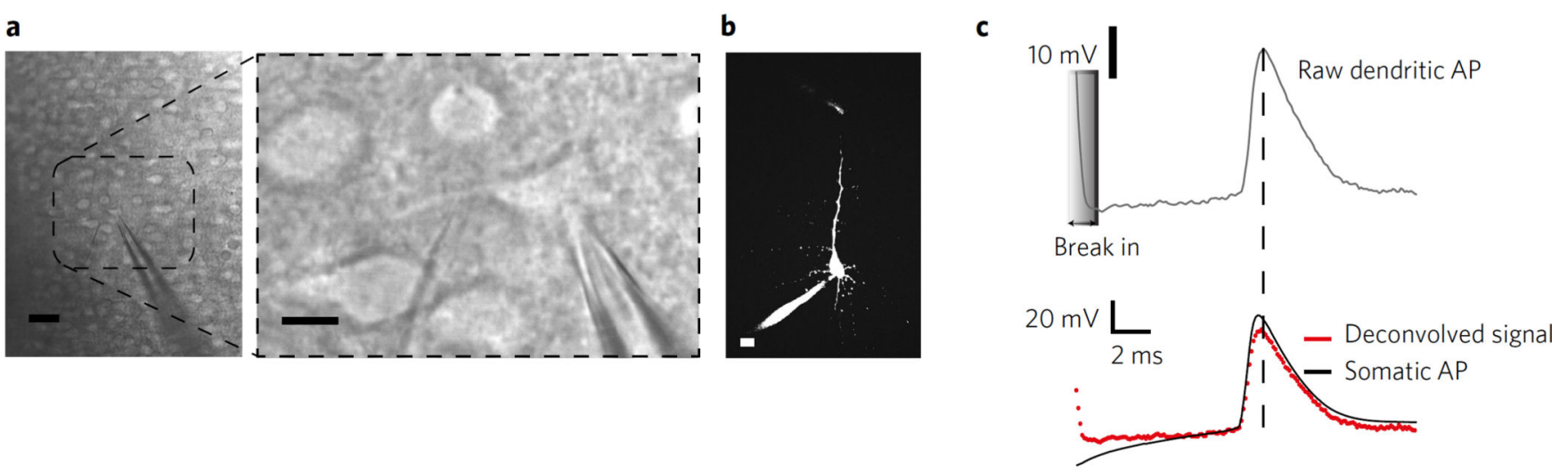

d
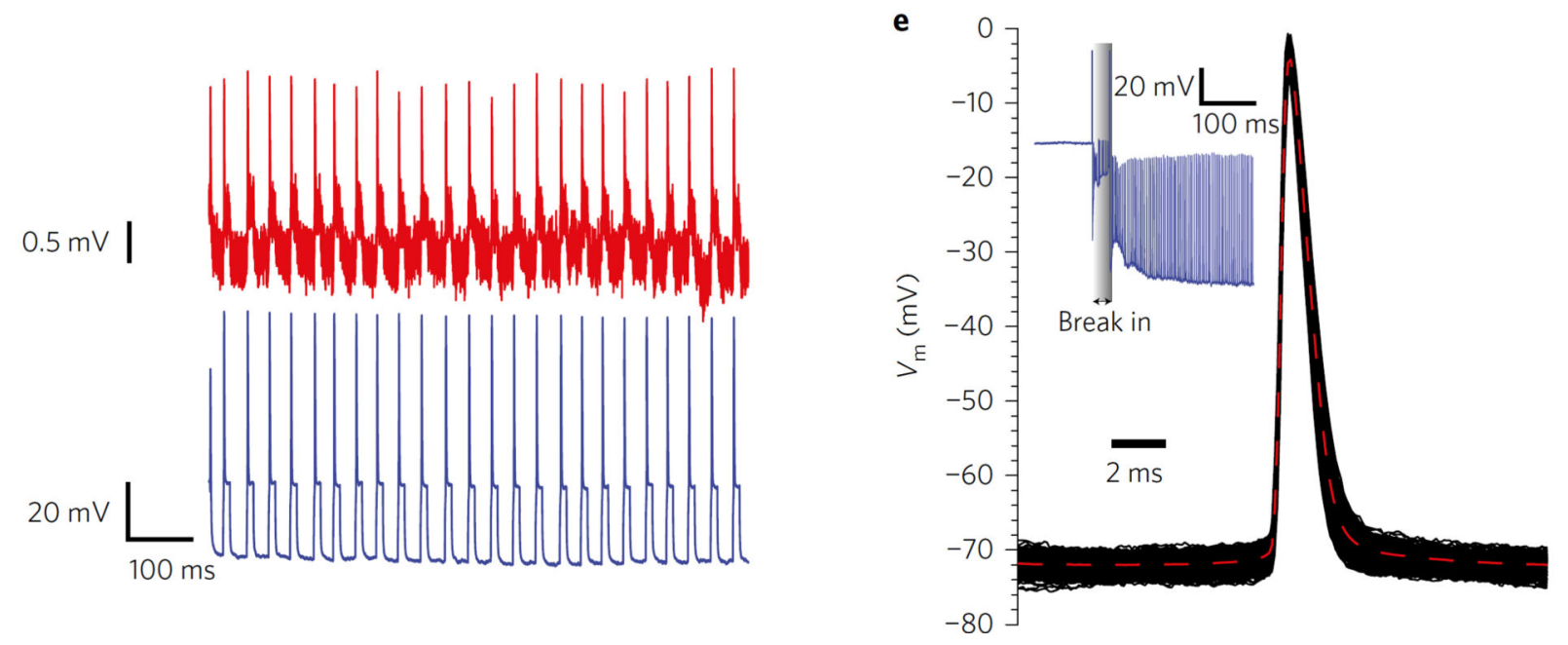

Figure 4. Nanopipette recordings from neocortical slices

a, Oblique illumination image of simultaneous somato-dendritic recordings in slices from layer-5 pyramidal neurons (left, large field of view, scale bar, $40 \mu \mathrm{m}$ ) and right (close up, scale bar, $10 \mu \mathrm{m}$ ). b. Two-photon image of simultaneous somato-dendritic recordings (scale bar, $10 \mu \mathrm{m})$. c, Deconvolved somato-dendritic recordings show full bAPs propagation up to $100 \mu \mathrm{m}$ from the soma. Dendritic recordings were initiated through electroporation-induced entry. d, Simultaneous somato-dendritic recordings (red-dendrite, blue-soma) show stable and repeatable time-locked bAPs registry. Dendritic recordings were initiated through electroporation. The formation of a stable $R_{\text {pore }}$ and $R_{\text {seal }}$ allowed prolonged (15-30 min) recordings. e, STA of raw somatic APs through mechanical impalement using nanopipettes (inset: break-in transient, blue). Note the RMP and amplitude of the AP revealing a high $R_{\text {shunt }}$. 


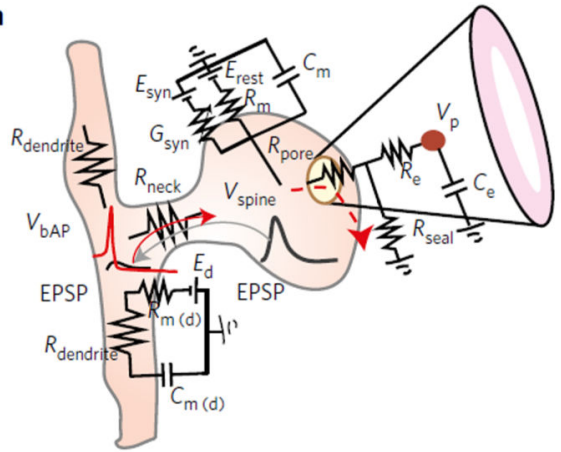

d

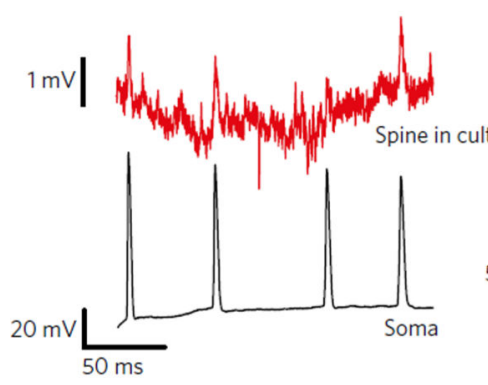

g

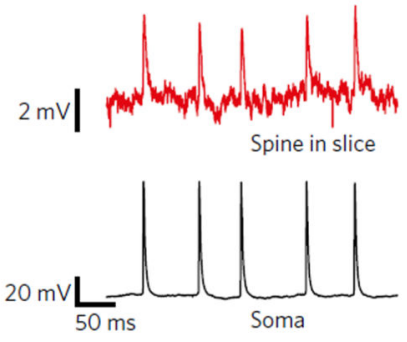

e

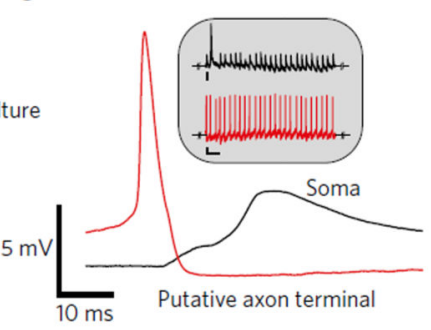

h

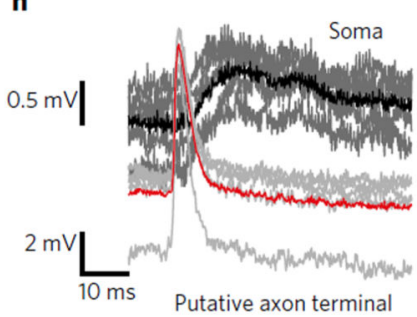

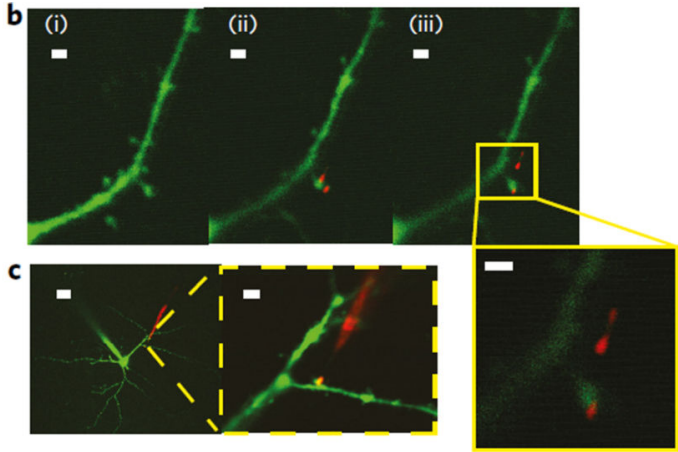

f

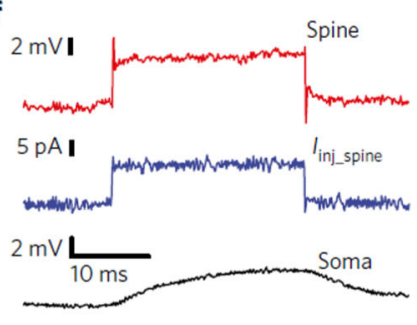

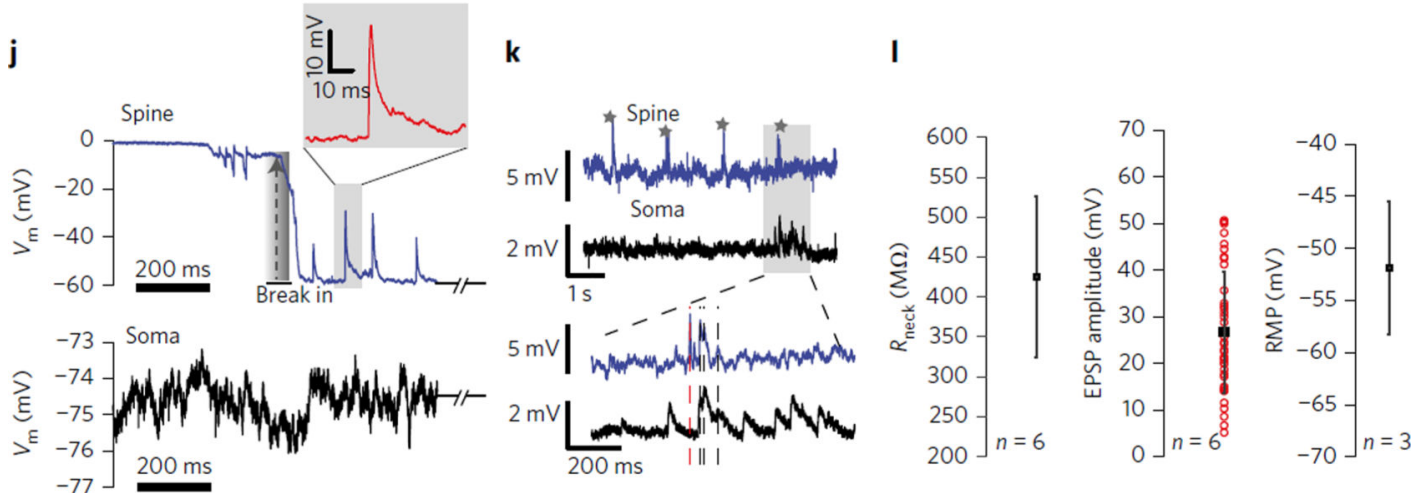

Figure 5. Nanopipette recordings in spines reveals electrical compartmentalization

a, Electrical equivalent circuit of the passive dendritic spine. $E_{\text {rest }}$, leak reversal potential;

$R_{\mathrm{m}}$, spine head passive membrane resistance; $C_{\mathrm{m}}$, spine head passive membrane capacitance; $E_{\mathrm{syn}}$, synaptic reversal potential; $g_{\mathrm{syn}}$, synaptic conductance; $R_{\text {pore }}$, pore resistance; $R_{\text {seal }}$, seal resistance; $R_{\text {neck }}$, neck resistance; $R_{\mathrm{e}}$, pipette resistance; $C_{\mathrm{e}}$, pipette capacitance; $R_{\text {dendrite }}$, dendritic resistance; $R_{\mathrm{m}(\mathrm{d})}$, dendrite passive membrane resistance; $C_{\mathrm{m}(\mathrm{d})}$, dendrite passive membrane capacitance; $E_{\mathrm{d}}$, dendrite reversal potential. bAPs invade the spine across the voltage divider formed by the parallel combination of $R_{\text {pore }} / / R_{\mathrm{m}} / / 1 / g_{\text {syn }}$ 
and $R_{\text {neck }}$. EPSPs in the spine head propagate into the dendrite across the divider formed between $R_{\text {neck }}$ and $R_{\text {dendrite }}$ b,c, Dual somato-spine recordings in cultures (b, scale bar, 1.5 $\mu \mathrm{m}$, panels (i)-(iii) and callout) and slices (c, scale bar, $20 \mu \mathrm{m}$ left, $3 \mu \mathrm{m}$, inset middle). Dendritic and spine nanopipette recordings were restricted to within $100 \mu \mathrm{m}$ from the soma to avoid space clamping and bAP attenuation. Note the QD (labelled red) desorption during nanopipette entry into the spine in the callout. $\mathbf{d}-\mathbf{i}$, Spine and pre-synaptic axon terminal recordings in cultures (d-f) and slices (g-i). d, bAPs invasion into spines. Somatic AP (bottom) and bAPs in the spine head (top) reveal that the nanopipette is capable of registering bAPs without the need for averaging. $\mathbf{e}$, STA of nanopipette recordings in the pre-synaptic axon terminal and post-synaptic soma. Note the characteristic 2-4 ms time delay between the pre-synaptic AP peak and onset of the post-synaptic EPSP (inset: raw data, scale bar, $2 \mathrm{mV}, 500 \mathrm{~ms}$ ). f, $15 \mathrm{pA}$ current injection into the spine head leads to a large potential change in the spine channel, but a filtered output at the soma. The voltage transient in the spine head is not clearly observable, which we attribute to shunting. g, bAPs registration in spines probed in slices. $\mathbf{h}$, STA of putative pre-synaptic terminal recordings in slice preparations. Note the very short delay between the putative pre-synaptic AP peak and the post-synaptic EPSP rise measured at the soma. i, STA of raw bAPs and somatic APs. Note the close fit in rise time and half width indicating the bAPs invade the spine with nearly no loss in temporal structure. $\mathbf{j}, \mathbf{k}$, EPSPs in spines. $\mathbf{j}$, Spontaneous break-in into the spine head (top) in cultures and corresponding somatic recordings (bottom). Note the large and fast rising spontaneous EPSPs registered in the spine that are completely filtered from the soma. The grey shaded region is zoomed in to show the typical rise and decay kinetics of the potential transient. k, Raw EPSPs in spines from slices (marked stars) are larger than somatic EPSPs ( $\sim 0.5 \mathrm{mV}$ flickers). Note the sudden registration of EPSPs in the soma (grey bar, bottom) that signify concomitant inputs from other spines. I, Deconvolved $R_{\text {neck }}$ (left), EPSP amplitudes (middle) and RMPs (right) in the spine head. The range of deconvolved EPSP amplitudes is in accordance with the spontaneous data from $\mathbf{j}$ (top). The range for $R_{\text {neck }}$ indicates that EPSPs that invade a low input resistance dendrite will be heavily attenuated, which is in line with data from $\mathbf{j}, \mathbf{k}$. 